\title{
Accelerated identification of Staphylococcus aureus from blood cultures by a modified fluorescence in situ hybridization procedure
}

Correspondence
Sven Poppert
poppert@bni-hamburg.de

Received 26 August 2009

Accepted 24 September 2009

\author{
Sven Poppert, ${ }^{1}$ Melanie Riecker, ${ }^{2}$ Nele Wellinghausen, ${ }^{2}$ \\ Hagen Frickmann ${ }^{3}$ and Andreas Essig ${ }^{2}$ \\ ${ }^{1}$ Bernhard Nocht Institute for Tropical Medicine, Hamburg, Germany \\ ${ }^{2}$ Institute of Medical Microbiology and Hygiene, University of Ulm, Ulm, Germany \\ ${ }^{3}$ Institute of Medical Microbiology, Virology and Hygiene, University of Rostock, Rostock, Germany
}

This study evaluated fluorescence in situ hybridization (FISH) for rapid identification of Staphylococcus aureus and coagulase-negative staphylococci (CoNS) directly from blood cultures. Initially, 360 blood cultures containing Gram-positive cocci were investigated by a previously described microwave-FISH procedure: 44/49 (89.8\%) S. aureus and 298/299 $(99.7 \%)$ CoNS were correctly identified. Because FISH proved useful and reliable but handling was found to be inconvenient, the method was modified by employing a recently developed slide chamber. This reduced the time required from 60 to $30 \mathrm{~min}$. The simplified execution allowed integration of the method into the workflow of a routine laboratory without difficulty. The modified method proved to be highly reliable, identifying $37 / 37$ (100\%) S. aureus and 169/172 (98.2\%) CoNS directly from blood cultures.

\section{INTRODUCTION}

Staphylococci are the bacteria isolated most frequently from blood cultures. The fastest possible identification of Staphylococcus aureus and its differentiation from coagulase-negative staphylococci (CoNS) are essential for optimal treatment of infected patients (Ruimy et al., 2008; Weinstein, 2003). Conventional phenotypic tests are not sufficiently reliable when applied directly to blood cultures (Chapin \& Musgnug, 2003; Mehta et al., 2009; Speers et al., 1998). A wide variety of molecular methods are available, but most are rather complex (Mehta et al., 2009; Ruimy et al., 2008). A relatively easy and costeffective molecular method for the identification of Staphylococcus aureus from blood cultures is fluorescence in situ hybridization (FISH) with either oligonucleotides of DNA (Gescher et al., 2008; Jansen et al., 2000; Kempf et al., 2000; Peters et al., 2006; Tavares et al., 2008) or peptide nucleic acid (PNA) (Chapin \& Musgnug, 2003; Forrest et al., 2006; González et al., 2004; Hartmann et al., 2005; Hensley et al., 2009; Ly et al., 2008; Oliveira et al., 2002, 2003). FISH allows species-specific staining of bacteria on a microscope slide by hybridization with fluorescently labelled oligonucleotide probes complementary to unique target sites on the rRNA gene. Compared with PNA-FISH, DNA-FISH is less standardized and less well evaluated for

Abbreviations: CoNS, coagulase-negative staphylococci; FISH, fluorescence in situ hybridization; PNA, peptide nucleic acid. the identification of Staphylococcus aureus. DNA probes are, however, less expensive and less prone to non-specific binding (Moter \& Gobel, 2000; Oliveira et al., 2002). Recently, a DNA-FISH procedure was developed that uses a microwave oven and allows a result to be obtained in $1 \mathrm{~h}$, about $2 \mathrm{~h}$ faster than conventional PNA- or DNA-FISH (Peters et al., 2006). This procedure has so far been tested with only 100 blood cultures that contained various bacteria, including in total just seven isolates of Staphylococcus aureus (Peters et al., 2006). We therefore investigated 360 blood cultures containing Gram-positive cocci using the microwave procedure. In order to improve the ease and speed of the method further, we modified the procedure by employing a recently developed slide chamber. This modified FISH procedure was evaluated on 220 additional blood cultures in the setting of a routine diagnostic laboratory.

\section{METHODS}

Reference strains. The following reference strains were obtained from the American Type Culture Collection: Bacillus subtilis ATCC 6633; Enterococcus avium ATCC 14025; Enterococcus casseliflavus ATCC 12755, 25788; Enterococcus durans ATCC 19432; Enterococcus faecalis ATCC 29212, ATCC 19433; Enterococcus faecium ATCC 19434, ATCC 51559; Enterococcus gallinarum ATCC 700425; Enterococcus hirae ATCC 8043; Enterococcus mundtii ATCC 43186; Enterococcus raffinosus ATCC 49427; Enterococcus saccharolyticus ATCC 43076; Micrococcus luteus ATCC 7468; Staphylococcus aureus ATCC 25923, ATCC 29213, ATCC 43300; Staphylococcus auricularis 
ATCC 33753; Staphylococcus epidermidis ATCC 12228; Staphylococcus hyicus ATCC 11249; Staphylococcus lugdunensis ATCC 49576; Staphylococcus saprophyticus ATCC 15305; Streptococcus agalactiae ATCC 13813; Streptococcus intermedius ATCC 27335; Streptococcus pneumoniae ATCC 49619; Streptococcus pyogenes ATCC 12344.

Clinical isolates. Clinical isolates were grown from blood cultures (BACTEC 9240; Bactec PLUS aerobic/F medium; Bactec PLUS anaerobic F; and Bactec PLUS paediatric; BD at the Institute of Medical Microbiology and Hygiene, University of Ulm, Germany). The isolates were characterized phenotypically by Gram-staining; colony morphology; haemolysis on blood agar; catalase reaction; growth on agar with trehalose, mannitol and phenolphthalein diphosphate; and the presence of clumping factor (Slidex; bioMérieux). Ambiguous isolates were confirmed by RAPIDEC Staph or API Staph ID 32 (bioMérieux). When biochemical identification was impossible or discrepancies were seen with the FISH result $(n=6)$, sequencing of the $16 \mathrm{~S}$ rRNA gene was performed as described previously (Hiraishi, 1992).

FISH. For FISH, previously published probes targeting either the entire Staphylococcus genus (Sta 16S697: 5'-TCCTCCATATCTCTGCGC-3') or Staphylococcus aureus specifically (Sau 16S69: 5' GAAGCAAGCTTCTCGTCCG-3') were directly $5^{\prime}$-labelled with the red sulfoindocyanine dye Cy3 (Thermo Fisher Scientific) (Kempf et al., 2000). These were used in combination with a green-fluorescing FAM-labelled eubacterial probe, EUB338, targeting almost all bacteria (Amann et al., 1990). CoNS were identified as organisms that stained with the staphylococcus probe but not with the Staphylococcus aureus probe.

For preparation of FISH slides from subcultured bacterial colonies, suspensions of bacteria in $0.9 \%$ saline were applied to the wells of an eight-well glass slide (Menzel), air dried and fixed in methanol for $10 \mathrm{~min}$. For FISH from blood cultures using the microwave FISH procedure, the blood culture medium was centrifuged at $130 \mathrm{~g}$ for $10 \mathrm{~min}$ to remove erythrocytes. The supernatant was centrifuged again at $1800 \mathrm{~g}$ for $5 \mathrm{~min}$. The pellet was suspended in $0.9 \%$ saline and applied to an eight-well glass slide. After air drying, the slides were fixed in pure methanol for $10 \mathrm{~min}$.

Permeabilization was conducted by adding $6 \mu \mathrm{l}$ lysis reagent, comprising lysozyme $\left(2 \mathrm{mg} \mathrm{ml}^{-1}\right.$; Sigma-Aldrich) and lysostaphin $\left(100 \mu \mathrm{g} \mathrm{ml}^{-1}\right.$; Sigma-Aldrich) in $10 \mathrm{mM}$ Tris/HCl ( $\mathrm{pH} \mathrm{8)}$, to each well of the eight-well slides. The slides were incubated at $37{ }^{\circ} \mathrm{C}$ for $15 \mathrm{~min}$ for staphylococci or for $1 \mathrm{~min}$ for enterococci and streptococci. The reaction was stopped by incubation for $3 \mathrm{~min}$ in methanol.

The FISH reaction was performed at a formamide concentration of $30 \%$ in a microwave oven (Sharp R208; SeaPro), as described recently (Peters et al., 2006).

Modified FISH. For the modified FISH procedure, blood culture medium $(0.3 \mathrm{ml})$ was not centrifuged but was diluted with $5 \mathrm{ml}$ PBS. A $10 \mu \mathrm{l}$ sample was applied directly onto eight-well slides, air dried and fixed with methanol for $10 \mathrm{~min}$. To save time, samples were prepared immediately from all blood cultures that were flagged positive. Only samples from blood cultures that later showed Gram-positive cocci in the Gram stain were investigated by FISH. Permeabilization was conducted by adding $10 \mu \mathrm{l}$ lysis reagent as above and incubating the slide for $5 \mathrm{~min}$ at $46{ }^{\circ} \mathrm{C}$ in a recently developed slide block (ThermoStat; Eppendorf). Hybridization was carried out in the same slide block at $46{ }^{\circ} \mathrm{C}$ for $10 \mathrm{~min}$. The washing step was performed in $50 \mathrm{ml}$ tubes in a thermoblock at $48{ }^{\circ} \mathrm{C}$ for $5 \mathrm{~min}$ with gentle shaking (Thermomixer; Eppendorf).

\section{RESULTS AND DISCUSSION}

\section{Evaluation of microwave-FISH}

In total, 360 blood cultures that showed Gram-positive cocci with a typical staphylococcus morphology in the Gram stain were investigated by microwave-FISH (Table 1). Only one sample per patient and only pure cultures were used for data analysis. FISH correctly identified 44/49 Staphylococcus aureus isolates, 298/299 CoNS and all 12 non-staphylococcal species (Table 1). For five samples with Staphylococcus aureus, no result was achieved directly from blood culture, because no bacteria were found on the slides and the control with the eubacterial probe was accordingly negative. One Staphylococcus epidermidis isolate was initially misidentified as a non-staphylococcus species because of a false-negative staining with the staphylococcus probe. All strains were correctly identified when FISH was repeated from subcultures. Three blood culture isolates had been identified as Staphylococcus aureus by phenotypic

Table 1. Identification of staphylococci directly from blood cultures

\begin{tabular}{|c|c|c|c|c|c|}
\hline Method & Staphylococcus aureus & CoNS & Micrococcus spp. & Rotia spp. & Others ${ }^{\star}$ \\
\hline \multicolumn{6}{|l|}{ Microwave procedure } \\
\hline Isolates in total & 49 & 299 & 10 & 1 & 1 \\
\hline Correct $\dagger$ & $44(89.7 \%)$ & $298(99.7 \%)$ & $10(100 \%)$ & $1(100 \%)$ & $1(100 \%)$ \\
\hline Without direct FISH result & $5(10.2 \%)$ & - & - & - & - \\
\hline False & - & $1(0.3 \%)$ & - & - & - \\
\hline \multicolumn{6}{|l|}{ Modified procedure } \\
\hline Isolates in total & 37 & 172 & 4 & 1 & 6 \\
\hline Correct $\dagger$ & $37(100 \%)$ & $169(98.2 \%)$ & $4(100 \%)$ & $1(100 \%)$ & $4(66.7 \%)$ \\
\hline Without direct FISH result & - & $2(1.2 \%)$ & - & - & - \\
\hline False & - & $1(0.6)$ & - & - & $2(33.3 \%)$ \\
\hline
\end{tabular}

${ }^{\star}$ Comprising Streptococcus sp. (one strain), Enterococcus spp. (five strains) and Kocuria sp. (one strain).

$\dagger$ The FISH assay allows identification to the species level only for Staphylococcus aureus. All other bacteria were classified either as CoNS or as nonstaphylococci. 
methods but were identified as CoNS by FISH. Sequencing revealed these strains as Staphylococcus hominis (two strains) and Staphylococcus haemolyticus (one strain). One Kocuria species had been misidentified as CoNS by phenotypic methods, but was correctly recognized as a non-staphylococcus species by FISH.

DNA-FISH using the microwave procedure thus proved to be highly reliable. However, application of the method in a routine laboratory was hampered by the need for sophisticated preparation steps and rather complicated handling of the microwave oven, which required preheating and complex calibration.

\section{Development and evaluation of a modified FISH procedure}

A simplified and even faster FISH procedure was therefore developed. The effects of the modifications were tested using subcultures of Staphylococcus aureus (ATCC 29213) and spiked blood cultures (data not shown). The different incubation times were reduced until the results visibly deteriorated, but for the final protocol, timing was increased to provide a safety margin. The centrifugation steps were omitted in order to save time and to improve the recovery of Staphylococcus aureus, on the assumption that Staphylococcus aureus tends to form clusters that may be lost with the erythrocytes during spinning. The staphylococcus cell walls were permeabilized for $5 \mathrm{~min}$ at $46{ }^{\circ} \mathrm{C}$ in a recently developed slide chamber instead of for $15 \mathrm{~min}$ at $37^{\circ} \mathrm{C}$ in an incubator. Using the same slide chamber for the hybridization allowed shortening of this step from 18 to $10 \mathrm{~min}$, because heat transmission in the slide block is even faster than in the microwave. The washing step was conducted in $50 \mathrm{ml}$ tubes by gentle shaking in a Thermomixer, which allowed shortening of the washing step from 14 to $5 \mathrm{~min}$. The total required time was thereby reduced from approximately $60 \mathrm{~min}$ to only $30 \mathrm{~min}$, and the whole procedure was considerably more convenient.

The modified FISH procedure was evaluated using 27 reference strains as listed in Methods and six clinical isolates of E. faecium, Streptococcus bovis, Streptococcus gallolyticus, Streptococcus gordonii, Streptococcus mitis and Streptococcus oralis. FISH produced correct results with 32/ 33 tested bacteria. Unfortunately, the Bacillus stained clearly positive with the general Staphylococcus probe and weakly positive with the Staphylococcus aureus probe. These cross-reactions also occurred when FISH was performed in the classic way in an incubator. Fortunately, these crossreactions do not impair the value of FISH for the identification of staphylococci in blood cultures, because bacilli, being Gram-positive rods, cannot be confused with staphylococci in a Gram stain.

In the next step, the applicability and reliability of the modified FISH procedure in a routine setting was tested using additional blood cultures showing Gram-positive cocci in the Gram stain. First, 55 blood cultures comprising 48 CoNS and seven Staphylococcus aureus were investigated using both FISH procedures. In this set, both procedures achieved correct results in all cases except that both procedures missed one CoNS. In these cases, no result was achieved because no bacteria were detected on the slide with the failed procedure, and in both cases a correct result was obtained with the other procedure.

After this successful pre-evaluation, the modified method was integrated into the workflow of the diagnostic laboratory and executed by routine personnel without difficulties. In total, 165 additional blood cultures were investigated, meaning that, overall, 220 blood cultures were examined with the modified method. Of these, all 37 Staphylococcus aureus and 169/172 CoNS were correctly diagnosed (Table 1). Two enterococci were misidentified as CoNS and as Staphylococcus aureus. This was probably caused by technical mistakes by less-experienced personnel, as correct results were achieved when FISH was repeated from pure culture, and the probes did not show any crossreaction with the reference strains of enterococci. In addition, the staphylococcus probe (Sta 16S697) had been tested, in the scope of a previous study, with 60 enterococci and streptococci without showing any cross-reaction (Wellinghausen et al., 2007, unpublished data). One Staphylococcus capitis isolate was misidentified as Staphylococcus aureus. In this single case, the Staphylococcus aureus probe showed some faint crossreaction when FISH was repeated from pure culture. In contrast to the microwave-FISH procedure, all samples with Staphylococcus aureus were clearly assessable and correctly identified by the modified procedure.

The modified FISH procedure thus showed high sensitivity and specificity. The short time to result represents a significant advantage in comparison with conventional DNA and PNA-FISH procedures, as well with PCR, as it makes simultaneous reporting of the findings of Gram staining and FISH possible. This allows quick initiation of optimal antibiotic therapy, leading to improved patient outcome and cost savings (Barenfanger et al., 1999; Forrest et al., 2006; Ly et al., 2008; Ruimy et al., 2008).

In conclusion, FISH proved to be highly reliable for rapid identification of staphylococci from blood cultures. The modifications to the method considerably facilitated and accelerated the application in a routine diagnostic setting.

\section{REFERENCES}

Amann, R. I., Binder, B. J., Olson, R. J., Chisholm, S. W., Devereux, R. \& Stahl, D. A. (1990). Combination of $16 \mathrm{~S}$ rRNA-targeted oligonucleotide probes with flow cytometry for analyzing mixed microbial populations. Appl Environ Microbiol 56, 1919-1925.

Barenfanger, J., Drake, C. \& Kacich, G. (1999). Clinical and financial benefits of rapid bacterial identification and antimicrobial susceptibility testing. J Clin Microbiol 37, 1415-1418. 
Chapin, K. \& Musgnug, M. (2003). Evaluation of three rapid methods for the direct identification of Staphylococcus aureus from positive blood cultures. J Clin Microbiol 41, 4324-4327.

Forrest, G. N., Mehta, S., Weekes, E., Lincalis, D. P., Johnson, J. K. \& Venezia, R. A. (2006). Impact of rapid in situ hybridization testing on coagulase-negative staphylococci positive blood cultures. J Antimicrob Chemother 58, 154-158.

Gescher, D. M., Kovacevic, D., Schmiedel, D., Siemoneit, S., Mallmann, C., Halle, E., Göbel, U. B. \& Moter, A. (2008). Fluorescence in situ hybridisation (FISH) accelerates identification of Gram-positive cocci in positive blood cultures. Int J Antimicrob Agents 32 (Suppl. 1), S51-S59.

González, V., Padilla, E., Gimenez, M., Vilaplana, C., Pérez, A., Fernández, G., Quesada, M. D., Pallarés, M. A. \& Ausina, V. (2004). Rapid diagnosis of Staphylococcus aureus bacteremia using $S$. aureus PNA FISH. Eur J Clin Microbiol Infect Dis 23, 396-398.

Hartmann, H., Stender, H., Schafer, A., Autenrieth, I. B. \& Kempf, V. A. (2005). Rapid identification of Staphylococcus aureus in blood cultures by a combination of fluorescence in situ hybridization using peptide nucleic acid probes and flow cytometry. J Clin Microbiol 43, 4855-4857.

Hensley, D. M., Tapia, R. \& Encina, Y. (2009). An evaluation of the AdvanDx Staphylococcus aureus/CNS PNA FISH assay. Clin Lab Sci 22, 30-33.

Hiraishi, A. (1992). Direct automated sequencing of $16 \mathrm{~S}$ rDNA amplified by polymerase chain reaction from bacterial cultures without DNA purification. Lett Appl Microbiol 15, 210-213.

Jansen, G. J., Mooibroek, M., Idema, J., Harmsen, H. J., Welling, G. W. \& Degener, J. E. (2000). Rapid identification of bacteria in blood cultures by using fluorescently labeled oligonucleotide probes. J Clin Microbiol 38, 814-817.

Kempf, V. A., Trebesius, K. \& Autenrieth, I. B. (2000). Fluorescent in situ hybridization allows rapid identification of microorganisms in blood cultures. J Clin Microbiol 38, 830-838.

Ly, T., Gulia, J., Pyrgos, V., Waga, M. \& Shoham, S. (2008). Impact upon clinical outcomes of translation of PNA FISH-generated laboratory data from the clinical microbiology bench to bedside in real time. Ther Clin Risk Manag 4, 637-640.
Mehta, M. S., Paule, S. M., Thomson, R. B., Kaul, K. L. \& Peterson, L. R. (2009). Identification of Staphylococcus species directly from positive blood culture broth by use of molecular and conventional methods. J Clin Microbiol 47, 1082-1086.

Moter, A. \& Göbel, U. B. (2000). Fluorescence in situ hybridization (FISH) for direct visualization of microorganisms. J Microbiol Methods 41, 85-112.

Oliveira, K., Procop, G. W., Wilson, D., Coull, J. \& Stender, H. (2002). Rapid identification of Staphylococcus aureus directly from blood cultures by fluorescence in situ hybridization with peptide nucleic acid probes. J Clin Microbiol 40, 247-251.

Oliveira, K., Brecher, S. M., Durbin, A., Shapiro, D. S., Schwartz, D. R., De Girolami, P. C., Dakos, J., Procop, G. W., Wilson, D. \& other authors (2003). Direct identification of Staphylococcus aureus from positive blood culture bottles. J Clin Microbiol 41, 889-891.

Peters, R. P., van Agtmael, M. A., Simoons-Smit, A. M., Danner, S. A., Vandenbroucke-Grauls, C. M. \& Savelkoul, P. H. (2006). Rapid identification of pathogens in blood cultures with a modified fluorescence in situ hybridization assay. J Clin Microbiol 44, 41864188.

Ruimy, R., Dos-Santos, M., Raskine, L., Bert, F., Masson, R., Elbaz, S., Bonnal, C., Lucet, J. C., Lefort, A. \& other authors (2008). Accuracy and potential usefulness of triplex real-time PCR for improving antibiotic treatment of patients with blood cultures showing clustered Gram-positive cocci on direct smears. J Clin Microbiol 46, 2045-2051.

Speers, D. J., Olma, T. R. \& Gilbert, G. L. (1998). Evaluation of four methods for rapid identification of Staphylococcus aureus from blood cultures. J Clin Microbiol 36, 1032-1034.

Tavares, A., Inacio, J., Melo-Cristino, J. \& Couto, I. (2008). Use of fluorescence in situ hybridization for rapid identification of staphylococci in blood culture samples collected in a Portuguese hospital. J Clin Microbiol 46, 3097-3100.

Weinstein, M. P. (2003). Blood culture contamination: persisting problems and partial progress. J Clin Microbiol 41, 2275-2278.

Wellinghausen, N., Bartel, M., Essig, A. \& Poppert, S. (2007). Rapid identification of clinically relevant Enterococcus species by fluorescence in situ hybridization. J Clin Microbiol 45, 3424-3426. 